\title{
RESEARCH
}

Open Access

\section{The outcome of COVID-19 among the geriatric age group in African countries: protocol for a systematic review and meta- analysis}

Degena Bahrey Tadesse ${ }^{1 *}$ D, Shishay Wahdey², Melaku Negash', Ebud Ayele ${ }^{3}$, Teklehaimanot Gereziher Haile ${ }^{4}$, Kbrom Gemechu Kiros ${ }^{5}$, Yohannes Ashebir Tesfamichael ${ }^{6}$ and Kiros Belay Gebrekidan ${ }^{7}$

\begin{abstract}
Background: According to the World Health Organization (WHO), the outbreak of coronavirus disease in 2019 (COVID-19) has been declared as a pandemic and public health emergency that infected more than 5 million people worldwide at the time of writing this protocol. Strong evidence for the outcome of COVID-19 among the geriatric age group has not been published in Africa. Therefore, this protocol will be served as a guideline to conduct a systematic review and meta-analysis of the outcome of COVID-19 among the geriatric age group in Africa.

Methods: Published and unpublished studies on the outcome of COVID-19 among the geriatric age group in Africa and written in any language will be included. Databases (PubMed / MEDLINE, Google Scholar, Google, EMBASE, Web of Science, Microsoft Academic, WHO COVID-19 database, Cochran Library, Africa Wide Knowledge, and Africa Index Medicus) from March to August 2020 will be searched. Two independent reviewers will select, screen, extract data, and assess the risk of bias. The proportion will be measured using a random-effects model. Subgroup analysis will be conducted to manage heterogeneity. The presence of publication bias will be assessed using Egger's test and visual inspection of the funnel plots. This systematic and meta-analysis review protocol will be reported per the PRISMA-P guidelines.
\end{abstract}

Conclusion: This systematic review and meta-analysis protocol will be expected to quantify the outcome of COVID19 among the geriatric age group in Africa.

Systematic review registration: This protocol was submitted for registration with the International Prospective Register of Systematic Reviews (PROSPERO) in April 2020 and accepted with the registration number: (https://www. crd.york.ac.uk/PROSPERO). CRD42020180600.

Keywords: Africa, COVID-19, Geriatric, Outcome

\footnotetext{
*Correspondence: shewitdege@gmail.com; degenabahrey@gmail.com

'Department of Adult Health Nursing, School of Nursing, Aksum University, Aksum, Ethiopia

Full list of author information is available at the end of the article
}

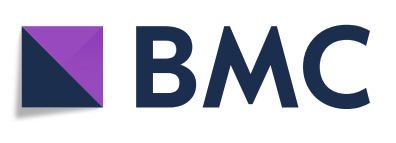

( ) The Author(s). 2020 Open Access This article is licensed under a Creative Commons Attribution 4.0 International License, which permits use, sharing, adaptation, distribution and reproduction in any medium or format, as long as you give appropriate credit to the original author(s) and the source, provide a link to the Creative Commons licence, and indicate if changes were made. The images or other third party material in this article are included in the article's Creative Commons licence, unless indicated otherwise in a credit line to the material. If material is not included in the article's Creative Commons licence and your intended use is not permitted by statutory regulation or exceeds the permitted use, you will need to obtain permission directly from the copyright holder. To view a copy of this licence, visit http://creativecommons.org/licenses/by/4.0/. The Creative Commons Public Domain Dedication waiver (http://creativecommons.org/publicdomain/zero/1.0/) applies to the data made available in this article, unless otherwise stated in a credit line to the data. 


\section{Background}

Coronavirus disease 2019 (abbreviated "COVID-19") is an infectious respiratory illness caused by a novel coronavirus, first identified in Wuhan, China, in December 2019. It is a large class of viruses that have been relatively widespread all across the world. The virus has low pathogenicity and high transmissibility capacity [1]. The Chinese authorities in Wuhan City, the capital of the province of Hubei, China, first announced this at the end of December 2019 [2-5]. After originating in Wuhan China last December 2019, COVID-19 has spread to at least 200 countries and region. The coronavirus disease 2019 (COVID-19) outbreak was declared a public health emergency of international concern by the World Health Organization (WHO) [6]. According to the WHO, the outbreak of coronavirus disease in 2019 (COVID-19) has been a pandemic that infected more than 5 million people at the time of writing this protocol and caused more than 324,000 deaths and 1.7 million recoveries worldwide within the 6 months duration [7]. Of the total cases, $15 \%$ develop severe disease, including pneumonia, and $5 \%$ become critically ill with respiratory failure, septic shock, and/or multi-organ failure [8].

The geriatric age groups were the most vulnerable age group and the Wuhan virus killing mostly the elderly. Of the 70 - to 79-year-olds infected with coronavirus there, $8 \%$ have died. Among the over-80s, the case fatality rate is $15 \%$ [9].

A modeling study in Africa, classifying African countries as having high risk with 13 top WHO highest priority (Egypt, Algeria, and South Africa), moderate risk (Nigeria, Ethiopia, Morocco, Sudan, Angola, Tanzania, Ghana, and Kenya) and all other countries had low to moderate importation risk. The fatality rate of COVID in north African countries expected to be $11.02 \%[10,11]$.

Generally speaking, African countries have poor health systems and this remains a source of concern, particularly in the event of an increase in outbreaks [12]. Due to this reason at the end of the pandemic, Africa might have some of the worst consequences of this COVID-19 pandemic.

In the WHO Africa region alone 83,000 to 190,000 people could die of COVID-19 and 29 million to 44 million could get infected in the first year of the pandemic if nt measurement will not be taken. The research, which is based on prediction modeling, looks at 47 countries in the WHO African Region with a total population of 1 million. There would be an estimation of 3.6 to 5.5 million COVID-19 hospitalizations, of those 82, 000 to 167,000 would be severe cases requiring oxygen, and 52,000 to 107,000 would be critical cases requiring breathing support [13].

There were different reports regarding the COVID-19 [14-16] but; there are no pooled results regarding the outcome of COVID-19 among the geriatric age group in
Africa. Therefore, this study protocol will be guided to conduct a systematic review and meta-analysis of the outcome of COVID-19 among the geriatric age group in Africa.

\section{Methods \\ Protocol registration}

This review is registered in PROSPERO International Prospective Register of Systematic Reviews (CRD42020180600) (https://www.crd.york.ac.uk/PROSPERO)) and reported according to Preferred Reporting Items for Systematic reviews and MetaAnalysis protocol (PRISMA-P) guidelines [17] (Table 1).

\section{Search strategy and data extraction}

The search strategy has been applied using Online Databases (PubMed / MEDLINE, Google Scholar, Google, EMBASE, Web of Science, Microsoft Academic, WHO COVID-19 database, Cochran Library, Africa Wide Knowledge, and Africa Index Medicus) from March to August 2020(Table 2). During the PROSPERO registration, a total of 10 articles were identified. The search terms which shall be used: "Wuhan coronavirus" OR "COVID-19" OR "novel coronavirus" OR "2019-nCoV" OR "coronavirus disease" OR "SARS-CoV-2" OR "SARS2" OR "severe acute respiratory syndrome coronavirus 2" OR" admission" OR" Burden" OR "Outcome". Other searching terms will be used "mortality" OR "prevalence" OR" incidence" OR" cardiovascular complications" OR" Geriatrics Age Group" OR" renal complications" "hematological complications of COVID-19" OR"prevalence of asymptomatic, mild, moderate and severe cases" OR "admission" ("number admitted to specialized units or intensive care units" OR" outcome" ("number of infected patients OR" "number of recoveries" OR" case fatality rate" OR "number of cured patients readmitted" OR "long term complications" such as chronic heart failure, cardiac arrhythmias, and recurrent thromboembolic diseases.

Searching results will be independently evaluated by two different reviewers. The literature search technique will be developed using the headings of the medical subject headings (Mesh), BOOLEAN (AND/OR) operator will be used.

The blinding will be maintained by using the Royyan that allows/ obligates each reviewer to work without knowing the other reviewer's choice. This review will be created using the metadata of the "COVID-19 Open Research Dataset" (https://pages.semanticscholar.org/coronavirus-research) (updated May 2020). We only uploaded the metadata (reference) file of $63 \mathrm{k}+$ coronavirus and COVID-19 research articles with links to PubMed, Microsoft Academic, and the WHO COVID-19 database of publications. We had to transform the metadata file (using https://github.com/rayyanqcri/CORD-19importer) to make it compatible with Rayyan. $r$. 
Table 1 PRISMA-P (Preferred Reporting Items for Systematic Review and Meta-Analysis Protocols) 2015 checklist: recommended items to address in a systematic review protocol

\begin{tabular}{lllll}
\hline Section/topic & $\begin{array}{l}\text { Item } \\
\text { No }\end{array}$ & Checklist item & $\begin{array}{l}\text { Information } \\
\text { reported }\end{array}$ & $\begin{array}{l}\text { Line } \\
\text { number(s) }\end{array}$ \\
\cline { 2 - 4 } & & Yes & No & Yes \\
\hline
\end{tabular}

Administrative Information

Title:

Identification

Update

Registration

Authors

Contact

Contributions

Amendments

Support

Sources

Sponsor

Role of sponsor/funder

Introduction

Rationale

Objectives

Methods

Eligibility criteria

Information sources

Search strategy

Study Records

Data management

Selection process

Data collection process
Specify the study characteristics (e.g., PICO, study design, setting, time frame) and report characteristics (e.g., years considered, language, publication status) to be used as criteria for eligibility for the review

9

mechanism(s) that will be used to manage records and data throughout the review electronic databases, contact with study authors, trial registers, or other grey literature sources) with planned dates of coverage

The present draft of the search strategy to be used for at least one electronic database, including planned limits, such that it could be repeated

State the process that will be used for selecting studies (e.g., two independent reviewers) through each phase of the review (i.e., screening, eligibility, and inclusion in meta-analysis)

11c Describe the planned method of extracting data from reports (e.g., piloting forms, done independently, in duplicate), and processes for obtaining and confirming 
Table 1 PRISMA-P (Preferred Reporting Items for Systematic Review and Meta-Analysis Protocols) 2015 checklist: recommended items to address in a systematic review protocol (Continued)

\begin{tabular}{|c|c|c|c|c|c|}
\hline \multirow[t]{2}{*}{ Section/topic } & \multirow[t]{2}{*}{$\begin{array}{l}\text { Item } \\
\text { No }\end{array}$} & \multirow[t]{2}{*}{ Checklist item } & \multicolumn{2}{|c|}{$\begin{array}{l}\text { Information } \\
\text { reported }\end{array}$} & \multirow[t]{2}{*}{$\begin{array}{l}\text { Line } \\
\text { number(s) }\end{array}$} \\
\hline & & & Yes & No & \\
\hline & & data from investigators & & & \\
\hline Data items & 12 & $\begin{array}{l}\text { List and define all variables for which data will be } \\
\text { sought (e.g., PICO items, funding sources), any pre- } \\
\text { planned data assumptions and simplifications }\end{array}$ & & & \\
\hline Outcomes and prioritization & 13 & $\begin{array}{l}\text { List and define all outcomes for which data will be } \\
\text { sought, including prioritization of main and additional } \\
\text { outcomes, with rationale }\end{array}$ & & & \\
\hline Risk of bias in individual studies & 14 & $\begin{array}{l}\text { Describe anticipated methods for assessing the risk } \\
\text { of bias of individual studies, including whether this } \\
\text { will be done at the outcome or study level, or both; } \\
\text { state how this information will be used in data synthesis }\end{array}$ & & & \\
\hline \multicolumn{6}{|l|}{ Data } \\
\hline \multirow[t]{4}{*}{ Synthesis } & $15 a$ & $\begin{array}{l}\text { Describe criteria under which study data will be } \\
\text { quantitatively synthesized }\end{array}$ & & & \\
\hline & $15 b$ & $\begin{array}{l}\text { If data are appropriate for quantitative synthesis, describe } \\
\text { planned summary measures, methods of handling data, } \\
\text { and methods of combining data from studies, including } \\
\text { any planned exploration of consistency (e.g., } I^{2}, \\
\text { Kendall's tau) }\end{array}$ & & & \\
\hline & $15 c$ & $\begin{array}{l}\text { Describe any proposed additional analyses (e.g., sensitivity } \\
\text { or subgroup analyses, meta-regression) }\end{array}$ & & & \\
\hline & $15 d$ & $\begin{array}{l}\text { If quantitative synthesis is not appropriate, describe the type } \\
\text { of summary planned }\end{array}$ & & & \\
\hline Meta-bias(es) & 16 & $\begin{array}{l}\text { Specify any planned assessment of meta-bias(es) (e.g., publication } \\
\text { bias across studies, selective reporting within studies) }\end{array}$ & & & \\
\hline Confidence in cumulative evidence & 17 & $\begin{array}{l}\text { Describe how the strength of the body of evidence will } \\
\text { be assessed (e.g., GRADE) }\end{array}$ & & & \\
\hline
\end{tabular}

\section{Selection and data collection process}

Data will be Extracted using the predefined standardized extraction form. Full texts for the eligible titles and/or abstracts including those where there is uncertainty will be obtained for further assessment on whether to include in the study or not. Where necessary, authors will be contacted for additional information to confirm eligibility of studies. The agreement between review authors will be measured using Cohen's $\mathrm{\kappa}$ statistic. Reasons for excluding articles will be recorded.

Where there is missing information, the corresponding author of the study will be contacted to request the missing information. A maximum of three emails will be sent to the corresponding author to request for additional information before excluding the study. For studies appearing in more than one published article, we will

Table 2 Searching strategy

\begin{tabular}{lllll}
\hline Serial number & Databases & $\begin{array}{l}\text { Number of the } \\
\text { article found }\end{array}$ & $\begin{array}{l}\text { Number of the } \\
\text { article included }\end{array}$ & $\begin{array}{l}\text { Number of } \\
\text { Excluded article }\end{array}$ \\
\hline 1 & PubMed & $\mathrm{n}=$ & $\begin{array}{l}\text { Reason for } \\
\text { exclusion }\end{array}$ \\
2 & Google Scholar & $\mathrm{n}=$ & $\mathrm{n}=$ & $\mathrm{n}=$ \\
3 & $\mathrm{n}=$ & $\mathrm{n}=$ & $\mathrm{n}=$ \\
4 & Web of Science & $\mathrm{n}=$ & $\mathrm{n}=$ & $\mathrm{n}=$ \\
5 & Cochran Library & $\mathrm{n}=$ & $\mathrm{n}=$ & $\mathrm{n}=$ \\
6 & Africa Wide Knowledge & $\mathrm{n}=$ & $\mathrm{n}=$ & $\mathrm{n}=$ \\
7 & Africa Index Medicus & $\mathrm{n}=$ & $\mathrm{n}=$ & $\mathrm{n}=$ \\
9 & Microsoft Academic & $\mathrm{n}=$ & $\mathrm{n}=$ & $\mathrm{n}=$ \\
\hline
\end{tabular}


consider the most recent, comprehensive, and with the largest sample size. For surveys appearing in one article with multiple surveys conducted at different time points, we shall treat each survey as a separate study. For multinational studies, data will be separated to show the estimate at the country level.

Data extracted comprised information about the month of publication, country, and design of the study, admission rate, burden, outcome, diagnostic criteria, comorbidity, COVID-19, mean age,ethnicity, sex (male proportion), signs and symptoms, complications, prevalence and/or incidence, and risk factors.

\section{Inclusion and exclusion criteria}

Studies presented as original articles studies that assessed outcomes from COVID-19 will be included.

\section{Types of studies}

Observational studies (including cross-sectional, casecontrol, and cohort) and the randomized controlled trial will be included. In the case of duplicate reports, the most comprehensive and up-to-date version will be taken into account.

\section{Participants}

All Patients from 65 years old or more, who are African residence and will be diagnosed as having COVID-19.

\section{Intervention(s)/exposure(s)}

Demographic, clinical, laboratory, management, and outcome data will be reviewed.

\section{Outcome}

Epidemiological data, mortality, and clinical outcomes of COVID-19. Establishing the clinical and epidemiological features, outcomes of COVID-19.

\section{Settings}

Hospital-based studies.

\section{Publication date}

March to August 2020.

\section{Language}

All published and unpublished papers (thesis, manuscript, pre-print pending to be published, the report from WHO, uCommunicable Diseases Control, United Nations, and health authorities in different African countries) without the restriction of language will include in our review.

\section{Method of diagnosis}

No restriction on methods of diagnosis but we will conduct subgroup analysis based on diagnostic tools. WHO interim guidance and /or any WHO-recommended diagnostic criteria will be considered [18] (Table 3).

\section{Exclusion criteria}

Studies that did not explain the criteria for the level COVID-19 outcome; studies that didn't state the number of patients with COVID-19 will be excluded. Studies not performed in humans, qualitative studies, studies that lack relevant data needed to compute the outcome will be excluded. Experimental studies, letters, reviews, commentaries, editorials, case reports, or case series will be not included.

\section{Quality assessment and risk of bias in individual studies}

To assess the risk of bias and quality of studies included in this review, a tool developed by Hoy et al. for prevalence studies will be used [19] The tool contains 11 items; items $1-4$ assess the external validity, $5-10$ assess the internal validity, and item 11 is a summary of the overall risk by the reviewer based on the responses of the above 10 items which are scored 1 if yes and 0 if no. Studies will be classified as having a low $(>8)$, moderate or high $(\leq 5)$ risk of bias. Additional file 1 shows this in more detail regarding the checklist of bias measurement on the observational study. For RCTs, we will use the

Table 3 Laboratory testing for coronavirus disease (COVID-19) in suspected human cases: interim guidance

\begin{tabular}{|c|c|c|}
\hline Test & Type of sample & Timing \\
\hline \multirow[t]{6}{*}{ Nucleic acid amplification tests (NAAT) } & Lower respiratory tract & \multirow{6}{*}{$\begin{array}{l}\text { Collect on presentation. } \\
\text { Possibly repeated sampling to monitor clearance. } \\
\text { Further research needed to determine effectiveness } \\
\text { and reliability of repeated sampling. }\end{array}$} \\
\hline & Sputum & \\
\hline & Aspirate & \\
\hline & Lavage & \\
\hline & Upper respiratory tract & \\
\hline & $\begin{array}{l}\text { Nasopharyngeal and Oropharyngeal swabs } \\
\text { Nasopharyngeal wash/nasopharyngeal aspirate. }\end{array}$ & \\
\hline Serology & $\begin{array}{l}\text { Serum for serological testing once validated } \\
\text { and available. }\end{array}$ & $\begin{array}{l}\text { Paired samples are necessary for confirmation with the } \\
\text { initial sample collected in the first week of illness and the } \\
\text { second ideally collected } 2-4 \text { weeks later (optimal timing } \\
\text { for convalescent sample needs to be established). }\end{array}$ \\
\hline
\end{tabular}


SPIRIT 2013 Checklist [20]. Additional file 2 shows this in more detail regarding the checklist of SPIRIT.

\section{Data management}

A tool has been developed a priori to conduct the searching strategy and this will help to guide the screening and selection process. The tool will be piloted and revised before data extraction begins. First the search results will be uploaded to EndNote software and then duplicates will be removed.

\section{Data items}

The extraction form will be includes data on general information, authors, month, country, and region, type of publication, study characteristics (study design, setting, sample size, response rate, mean or median age, or age range), the outcome.

\section{Outcomes}

The outcome of COVID - 19 among the geriatric age group in Africa.

\section{Data synthesis, analysis, and presentation}

The data will be analyzed using the R software; V.3.5.3. All analyses will be performed using a "metaprop" routine using $\mathrm{R}$ version 3.5.3 for Windows [18]. Results will be reported as proportions with corresponding $95 \%$ confidence intervals (CIs). Forest plots will be drawn to visualize the combined outcome of COVID-19 and the extent of statistical heterogeneity between studies. Statistical heterogeneity will be assessed using the 2 test on Cochrane's Q statistic, 20 and quantified by calculating the $\mathrm{I}^{2}$ statistic (with values of 25,50 , and $75 \%$ is representative of the low, medium, and high heterogeneity, respectively) [19]. There will be a clinical heterogeneity between studies included in this study. The random-effects will be used to estimate the overall pooled outcome of COVID-19 in Africa. The presence of publication bias will be assessed using Egger's test and funnel plots [20]. $P$-value $<0.10$ on the Egger's test will be considered statistically significant for publication bias. Moreover, other relevant findings will be summarized in a narrative format.

The crude numerators and denominators from the individual studies will be used to recalculate the studyspecific outcome of COVID-19. The outcome estimates will be summarized by African geographic regions.

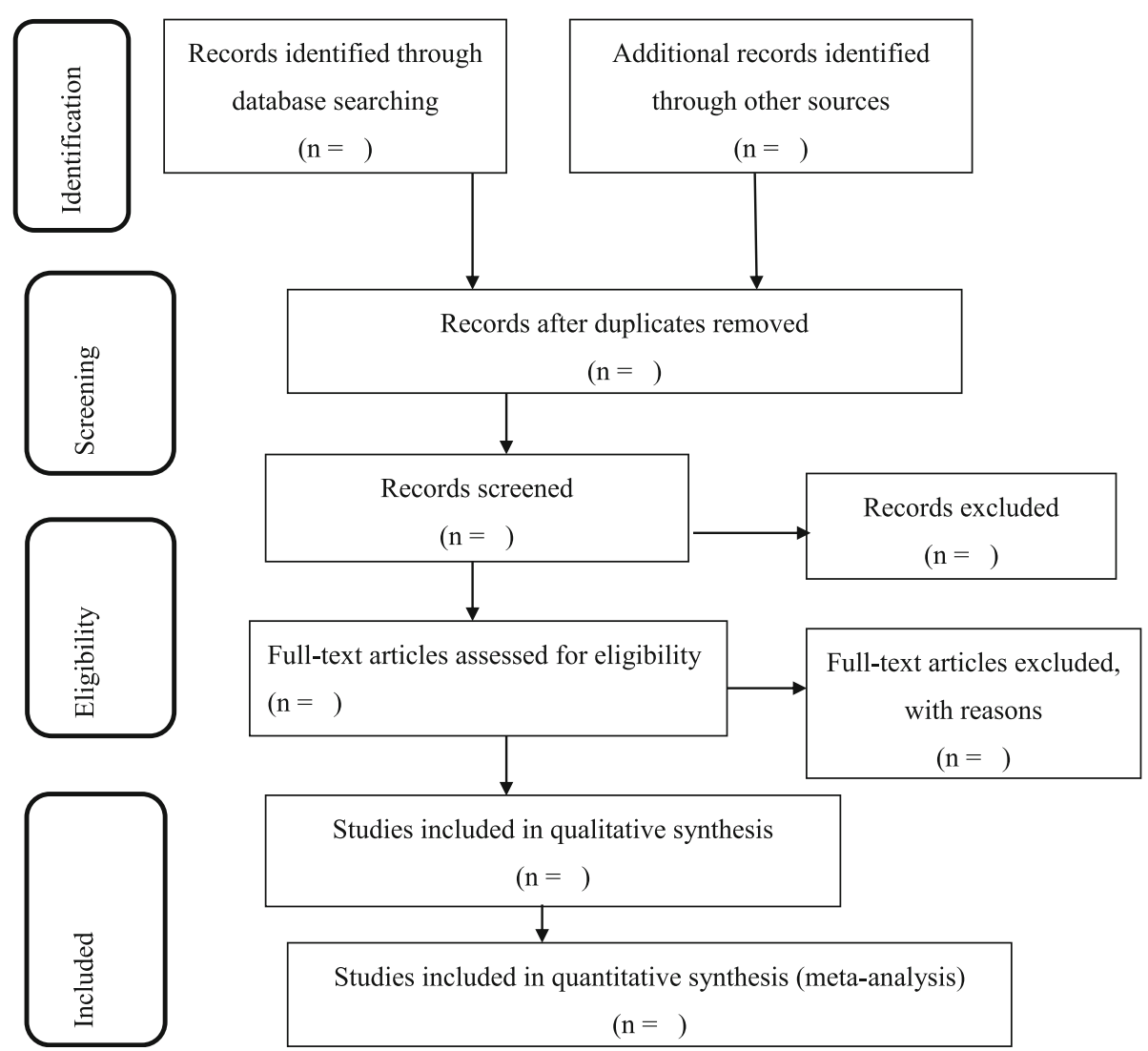

Fig. 1 Flow chart diagram will be shown the selection of articles for systemic review and meta-analysis of the outcome of COVID-19 among the geriatric age group in Africa 
A meta-analysis will be performed on variables that are similar across the included studies.

A subgroup analyses will be performed based on the countries where the study conducted, diagnostic method they used, based on their ethnic background (African origin and -non-African ethnic origin). The inter-rater agreements between the researchers involved in study selection and those involved in the identification of risk of bias will be assessed using $\kappa$ Cohen's coefficient.

\section{Discussion}

This systematic review and meta-analysis will be presented per the PRISMA checklist guidelines [14]. The PRISMA flow diagram will be used to record the different phases of the review process [14] (Fig. 1).

The summary will be used to display the data on the distribution of COVID-19 by variables of interest such as gender, residence, setting, and person-level characteristics. Funnel plots will be used to visualize publication bias of the included studies. Forest plots will be used to estimates the outcome of COVID-19 among the geriatric age group in Africa for the included studies as an overall pooled estimate for Africa. Results from this review will inform healthcare providers on the outcome of COVID -19 , hence providing evidence will bring the required changes needed in clinical practice and will support healthcare services in line with patients' needs. Findings from this review will be shared in conferences, peerreview journals, and social media platforms.

\section{Conclusion}

This systematic review and meta-analysis will be expected to quantify the outcome of COVID-19 among the geriatric age group in Africa to guide policies and interventions.

\section{Supplementary information}

Supplementary information accompanies this paper at https://doi.org/10. 1186/s40733-020-00064-8.

Additional file 1. Quality assessment checklist for prevalence studies (adapted from Hoy et al).

Additional file 2. SPIRIT 2013 Checklist: Recommended items to address in a randomized controlled trial.

\section{Abbreviations}

PRISMA-P: Preferred Reporting Items for Systematic reviews and MetaAnalysis protocol; WHO: World Health Organization; COVID-19: Coronavirus Disease in 2019

\section{Acknowledgments}

Not applicable.

\section{Authors' contributions}

DBT conceived and designed the initial study. All authors contributed to the conceptualization and design of the study. DBT drafted the initial protocol. DBT is the guarantor of the review. The author(s) read and approved the final manuscript.
Funding

Not applicable.

\section{Availability of data and materials}

The datasets used and/or analyses during the study will be presented within the manuscript and available from the corresponding author on reasonable request.

Ethics approval and consent to participate

Not applicable.

Consent for publication

Not applicable.

\section{Competing interests}

The authors declare that they have no competing interests.

\section{Author details}

'Department of Adult Health Nursing, School of Nursing, Aksum University, Aksum, Ethiopia. ${ }^{2}$ Department of Reproductive Health, School of Public Health, Mekelle University, Mekelle, Ethiopia. ${ }^{3}$ Department of Reproductive Health, School of Public Health, Aksum University, Aksum, Ethiopia.

${ }^{4}$ Department of Maternal and Neonatal Nursing, School of Nursing, Aksum University, Aksum, Ethiopia. ${ }^{5}$ Department of Adult Health Nursing, School of Nursing, Adigrat University, Adigrat, Ethiopia. ${ }^{6}$ Department of Pediatrics and Child Health Nursing, School of Nursing, Adigrat University, Adigrat, Ethiopia. ${ }^{7}$ Department of Adult Health Nursing, School of Nursing, Mekelle University, Mekelle, Ethiopia.

Received: 26 August 2020 Accepted: 5 October 2020

Published online: 08 October 2020

\section{References}

1. Jiang S, Shi Z, Shu Y, Song J, Gao GF, Tan W, Guo D. A distinct name is needed for the new coronavirus. Lancet (London, England). 2020; 395(10228):949.

2. Schoeman D, Fielding BC. Coronavirus envelope protein: current knowledge. Virol J. 2019;16(1):1-22.

3. Sohrabi $\mathrm{C}$, et al. World Health Organization declares global emergency: A review of the 2019 novel coronavirus (COVID-19). Int J Surgery. 2020;76:71-6. https://doi.org/10.1016/j.jisu.2020.02.034.

4. Mizumoto K, Kagaya K, Zarebski A, Chowell G. Estimating the asymptomatic proportion of coronavirus disease 2019 (COVID-19) cases on board the diamond princess cruise ship, Yokohama, Japan, 2020. Eurosurveillance. 2020;25(10):2000180.

5. Ho CS, Chee CY, Ho RC. Mental health strategies to combat the psychological impact of COVID-19 beyond paranoia and panic. Ann Acad Med Singap. 2020:49(1):1-3.

6. Al-Shamsi HO, Alhazzani W, Alhuraiji A, Coomes EA, Chemaly RF, Almuhanna M, Wolff RA, Ibrahim NK, Chua ML, Hotte SJ, Meyers BM. A practical approach to the management of cancer patients during the novel coronavirus disease 2019 (COVID-19) pandemic: an international collaborative group. Oncologist. 2020;25(6):e936.

7. Harries AD, Martinez L, Chakaya JM. Monitoring the COVID-19 pandemic in sub-Saharan Africa: focusing on health facility admissions and deaths. Int J Tuberc Lung Dis. 2020;24(5):550-2.

8. Geldsetzer P. Use of rapid online surveys to assess People's perceptions during infectious disease outbreaks: a cross-sectional survey on COVID-19. J Med Internet Res. 2020;22(4):e18790.

9. Gilbert M, Pullano G, Pinotti F, Valdano E, Poletto C, Boëlle PY, d'Ortenzio E, Yazdanpanah Y, Eholie SP, Altmann M, Gutierrez B. Preparedness and vulnerability of African countries against importations of COVID-19: a modelling study. Lancet. 2020;395(10227):871-7.

10. Daw MA, El-Bouzedi AH. Modelling the epidemic spread of COVID-19 virus infection in Northern African countries. Travel Med Infect Dis. 2020;35: 101671. https://doi.org/10.1016/j.tmaid.2020.101671.

11. Ohia C, Bakarey AS, Ahmad T. COVID-19 and Nigeria: putting the realities in context. Int J Infect Dis. 2020

12. Tadesse DB, Gebremeskel GG, Asefa GG, Abay M, Demoz GT. The burden, admission, and outcome of COVID-19 in Africa: protocol for a systematic review and meta-analysis. Emerging Microbes Infect. 2020;9(1):1372-8. 
13. Gupta M, Abdelmaksoud A, Jafferany M, Lotti T, Sadoughifar R, Goldust M. COVID-19 and economy. Dermatol Ther. 2020.

14. Hutton B, Catala-Lopez F, Moher D. The PRISMA statement extension for systematic reviews incorporating network meta-analysis: PRISMA-NMA. Med Clin (Barc). 2016;147(6):262-6.

15. Ferrari D, Motta A, Strollo M, Banfi G, Locatelli M. Routine blood tests as a potential diagnostic tool for COVID-19. Clin Chem Lab Med (CCLM). 2020; (ahead-of-print).

16. Hoy D, Brooks P, Woolf A, Blyth F, March L, Bain C, Baker P, Smith E, Buchbinder R. Assessing risk of bias in prevalence studies: modification of an existing tool and evidence of interrater agreement. J Clin Epidemiol. 2012;65(9):934-9.

17. Chan AW, Tetzlaff JM, Altman DG, Laupacis A, Gøtzsche PC, Krleža-Jerić K, Hróbjartsson A, Mann H, Dickersin K, Berlin JA, Doré CJ. SPIRIT 2013 statement: defining standard protocol items for clinical trials. Ann Intern Med. 2013;158(3):200-7.

18. Nyaga VN, Arbyn M, Aerts M. Metaprop: a Stata command to perform metaanalysis of binomial data. Archives Public Health. 2014;72(1):39.

19. Higgins JP, Thompson SG, Deeks JJ, Altman DG. Measuring inconsistency in meta-analyses. Bmj. 2003;327(7414):557-60.

20. Egger M, Smith GD, Schneider M, Minder C. Bias in meta-analysis detected by a simple, graphical test. Bmj. 1997;315(7109):629-34.

\section{Publisher's Note}

Springer Nature remains neutral with regard to jurisdictional claims in published maps and institutional affiliations.

Ready to submit your research? Choose BMC and benefit from:

- fast, convenient online submission

- thorough peer review by experienced researchers in your field

- rapid publication on acceptance

- support for research data, including large and complex data types

- gold Open Access which fosters wider collaboration and increased citations

- maximum visibility for your research: over $100 \mathrm{M}$ website views per year

At BMC, research is always in progress.

Learn more biomedcentral.com/submissions 